\title{
Article \\ Capability of Advanced Ultrasonic Inspection Technologies for Hydraulic Turbine Runners
}

\author{
Mohammad Ebrahim Bajgholi ${ }^{1, *(\mathbb{D})}$, Gilles Rousseau ${ }^{2}$, Martin Viens ${ }^{1}(\mathbb{D})$ and Denis Thibault ${ }^{2}$ \\ 1 École de Technologie Superieure, Montreal, QC H3C 1K3, Canada; martin.viens@etsmtl.ca \\ 2 Institut de Recherche d'Hydro-Québec (IREQ), Varennes, QC J3X 1S1, Canada; \\ rousseau.gillesa@hydroquebec.com (G.R.); thibault.denis@hydroquebec.com (D.T.) \\ * Correspondence: mohammadebrahim.bajgholi.1@ens.etsmtl.ca
}

check for updates

Citation: Bajgholi, M.E.; Rousseau, G.; Viens, M.; Thibault, D. Capability of Advanced Ultrasonic Inspection Technologies for Hydraulic Turbine Runners. Appl. Sci. 2021, 11, 4681. https://doi.org/10.3390/app11104681

Academic Editor:

Giuseppe Lacidogna

Received: 1 April 2021

Accepted: 20 April 2021

Published: 20 May 2021

Publisher's Note: MDPI stays neutral with regard to jurisdictional claims in published maps and institutional affiliations.

Copyright: (c) 2021 by the authors. Licensee MDPI, Basel, Switzerland. This article is an open access article distributed under the terms and conditions of the Creative Commons Attribution (CC BY) license (https:/ / creativecommons.org/licenses/by/ $4.0 /)$.

\begin{abstract}
This paper presents the results of a project aimed at evaluating the performance of ultrasonic techniques for detecting flaws in Francis turbine runners. This work is the first phase of a more ambitious program aimed at improving the reliability of inspection of critical areas in turbine runners. Francis runners may be utilized to supply power during peak periods, which means that they experience additional load stress associated with start and stop sequences. Inspection during manufacturing is then of paramount importance to remove as much as feasible all flaw initiation sites before the heat treatment. This phase one objective is to collect initial data on a simplified mock-up and then to compare the experimental ultrasonic data with the results of simulations performed by CIVA, a computer simulation package. The area of interest is the region with the highest stress between the blade and the web. A welded T-joint coupon made of UNS S41500 was manufactured to represent this high-stress area. During the FCAW welding process, ceramic beads were embedded in the weld to create discontinuities whose size is in the critical range to initiate a crack. Inspection of the material was carried out by various nondestructive testing (NDT) methods namely conventional pulse-echo, phased array, total focusing method (TFM). With these results, detection rates were obtained in order to compare the effectiveness of each method.
\end{abstract}

Keywords: nondestructive testing; defect detection; ultrasonic testing; phased-array ultrasonic testing; detection of rate; hydroelectric turbine runner

\section{Introduction}

In the province of Quebec, hydraulic turbines produce the vast majority of the electrical power [1]. Multi-pass flux-cored arc welding (FCAW), used for turbine runner joints, naturally generates certain discontinuities such as slag inclusions, lack of penetration, macro shrinkage, and thermal stress cracking. Welding discontinuities and dynamic stress concentration result in a higher failure probability in Francis turbine runners [2,3]. In the runner, the maximum stresses are located along the welds near the trailing edge of the blades. The critical weld region extends to approximately $300 \mathrm{~mm}$ from the trailing edge for both blade-to-crown and blade-to-web welds.

The Hydro-Quebec Research Institute (IREQ) has developed a life estimation model based on the distribution and size of structural discontinuities in turbines to ensure equipment reliability and control production costs. In this model, the probability of a cracking event at any given point in time depends on the joint probability of stress and defect being above the limit defined by the material properties [4]. Indeed, if discontinuities remain undetected, they might initiate fatigue cracks under dynamic, in-service stresses, leading to unplanned failure, which itself results in high repair costs and long downtime. It is desirable to supply the life estimation model with accurate data regarding the distribution and size of discontinuities present in the turbine to reduce the unplanned outage costs. This type of data is typically determined using NDT methods to detect, locate, and size flaws [2]. 
NDT is the development and application of technical methods to examine materials or components such that the pieces are not destroyed and their future usefulness and serviceability remain [5]. There is a wide range of NDT methods based on the physical principles of electromagnetic radiation, sound, and other inherent properties of the materials. The techniques selected should consider safety, economic, efficiency, and time factors [6]. Ultrasonic testing (UT) is a suitable method for detecting and sizing flaws embedded within or growing from inaccessible surfaces. Ultrasonic waves can propagate through thick solid parts [7]. Therefore, UT is suitable for flaws far beneath the inspection surface, unlike methods that can only detect defects on, or very close to, the inspection surface, e.g., Eddy current testing or methods that detect flaws connected to the inspection surface, such as visual methods and penetrant testing [8]. When defect assessment calculations require a value for how deep the defect has grown, ultrasonic testing is the primary desired method. It is used to detect various types of flaws, such as cracks and inclusions in a wide range of materials, compared to magnetic particle testing methods, which require the component to be ferromagnetic. Ultrasonic waves are inherently safe compared to radiography methods, which use ionizing radiation and thus require multiple protection types $[9,10]$.

The basic NDT methods, such as visual testing (VT), ultrasonic testing (UT), penetrant testing (PT), and radiography (RT), are usually used to inspect hydraulic turbine runners. However, there are difficulties involved in detecting internal flaws in complex geometries, such as the runner's weld joints. Thus, hydraulic turbine runners are generally inspected using different ultrasonic inspection methods [2,3,11-14]. Few studies have been performed on the inspection of hydraulic turbine runners with ultrasonic phased array. However, the published studies did not focus on the high-stress area, used artificial flaws embedded in a weld mockup, and did not consider the material's properties [11-13]. Boukani et al. [3,15] worked on the capability of different NDT methods and the effect of microstructure on the acoustic properties of steel grades widely used for the manufacturing of hydroelectric turbine runners. These studies concluded that phased array is a very effective technique.

The current study goes further by including the total focusing method (TFM), and compares conventional, phased array and TFM techniques in order to assess their performance in detecting flaws in the trailing edge of a runner blade. The work focuses on ultrasonic inspection techniques applied directly to the blade because they conform to current fabrication and in-service inspection methods. Simulations and experiments are used to identify the best practices to reliably inspect the blade high-stress regions.

This research, as part of a large project, focuses on the detection and characterization of flaws in the welded joints of hydroelectric turbine runners, as they are located in the most critical zones in terms of fatigue failure. Loading conditions and fatigue-related material properties have been previously studied in detail as two inputs of life estimation models $[4,16]$, while flaw characteristics are still fed into the developed models as assumptive third input values. The current research aims at providing IREQ with a flaw data reliability index (both measured and simulated) for the life estimation model.

\section{Experimental Procedure}

\subsection{Manufacturing Process Description}

Inspection of turbine runner welds is an essential aspect of electric-generator units' inservice reliability [4]. In-service inspection data from installed runners show that fabrication flaws are found in the weld joint near the blade's trailing edge. Flaws were discovered by applying a phased array ultrasonic technique using a small wedge to provide suitable contact on a curved surface and good volumetric coverage with an azimuthal focal law type. Typically, flaws are within $30 \mathrm{~mm}$ of the trailing edge and in the weld reinforcement that smooths the stresses at the blade's end. Some flaws were found isolated, but clusters of up to 7 indications were also observed. The presence of flaws at the edge of the blade in the area of high service stress is considered in the current investigation. This area is where the start and stop of welding occurs, and thus it is not surprising to observe in-situ point-like reflectors in the weld reinforcement. A T-joint sample was manufactured in martensitic 
stainless steel (UNS S41500) in accordance with the manufacturing requirements of turbine runners. The T-joint geometry often mimics a turbine runner's trailing edge, with both edges being $25 \mathrm{~mm}$ in thickness. The base plate is $60 \mathrm{~mm}$ thick and provides the same ultrasonic path for an inspection performed from the turbine web. The weld cap and blade ends are ground to obtain a smooth finish with a curved radius of $25 \mathrm{~mm}$. The preparation is a J-shape grove with a $3 \mathrm{~mm}$ gap between the web and blade. Welding was carried out using a multi-pass FCAW process and EC410NiMo electrodes (same ultrasonic properties as UNS S41500). The study selected the dimensions of the joint to match a typical turbine runner assembly.Typically, 34 passes are required to fill the joint.

\subsection{Flaw Size and Distribution}

For technical manufacturing constraints imposed by the small size of the flaws examined by the current study (e.g., size of flaws between $1 \mathrm{~mm}$ and $5 \mathrm{~mm}$ ), the scope is limited to inclusion flaws. Inclusions designate the incorporation, in the weld bead, of a foreign compound. Besides, the case of metal inclusions is studied to control the size of the flaws and the repeatability that are defined by the imprisonment of a foreign particle in the metal's mass. These flaws were relatively present in welded joints in laboratory samples that made inclusion a representative flaw for the current study. Spherical beads of a specific size are integrated into the weld fusion to simulate an inclusion. The choice of spherical shape is justified by limiting the external error factors that are linked to the defects' positioning and orientation.

To conduct the study, the requirement for precision on the defects' size, the obligation to respect an acceptable level of repeatability, and the flaws spherical shape guided in choosing ball bearings as the defect to be included. The extreme thermal conditions and the electric arc passage, during welding, close to the balls, forced the researchers to choose ceramic balls that were more resistant to extreme temperatures. This is because they interfered less with the heat-welding arc because of their non-conductive electrical properties.

The study considered three types of ceramic balls; alumina oxide balls $\left(\mathrm{Al}_{2} \mathrm{O}_{3}\right)$, zirconia balls $\left(\mathrm{ZrO}_{2}\right)$, and silicon nitride balls $\left(\mathrm{Si}_{3} \mathrm{~N}_{4}\right)$. In this regard, all three types of balls were tried to determine the best ones for the study. After a series of tests, the study found that the alumina oxide $\left(\mathrm{Al}_{2} \mathrm{O}_{3}\right)$ balls were to be avoided. This is because, despite the fact they offer a higher operating temperature compared to silicon nitride $\left(\mathrm{Si}_{3} \mathrm{~N}_{4}\right)$, they did not resist the electric arc passage and exploded [17]. Therefore, the study chose the silicon nitride balls because they were stronger and kept their original shape.

During welding, holes have been drilled in the weld pass to implement artificial flaws at known locations, as shown in Figure 1. Ceramic beads are inserted into these holes, and the holes are filled by welding. Thirty ceramic beads are distributed in the weld of the T-joint sample. They were of either 1, 2, 3, 4, or $5 \mathrm{~mm}$ in diameter (6 beads of each size). This range of discontinuity sizes reflects the critical range to initiate a fatigue crack. A three-millimeter diameter flaw has the critical size to develop a fatigue crack under cyclic loading from stress calculation. Beads are located within $75 \mathrm{~mm}$ from the edges of the sample in order to mimic the situation observed on in-service runners.

\subsection{Conventional Ultrasonic Testing (UT)}

Four experienced UT practitioners with CGSB (Canadian General Standards Board) level 2 or 3 certification in Ultrasonic Testing did inspect the T-joint sample. The study performed inspections as per the Canadian Standards Association (CSA) W59, Welded steel construction [18]. The study used a flat, $2.25 \mathrm{MHz} 1 / 2$ in round transducer mounted on a refracting wedge to produce a refracted shear wave beam at either $60^{\circ}$ or $70^{\circ}$ from normal. The conventional ultrasonic testing inspection was carried out using Olympus Epoch 600 and Krautkramer USN 58L Ultrasonic Flaw Detectors, as shown in Figure 2. All indications, rejectable or not, were to be reported. 


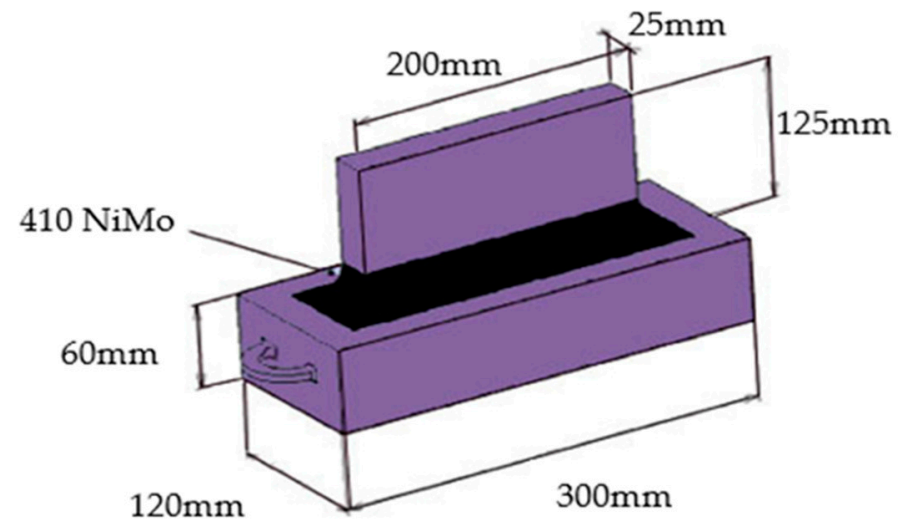

(a) Dimension of T-joint sample

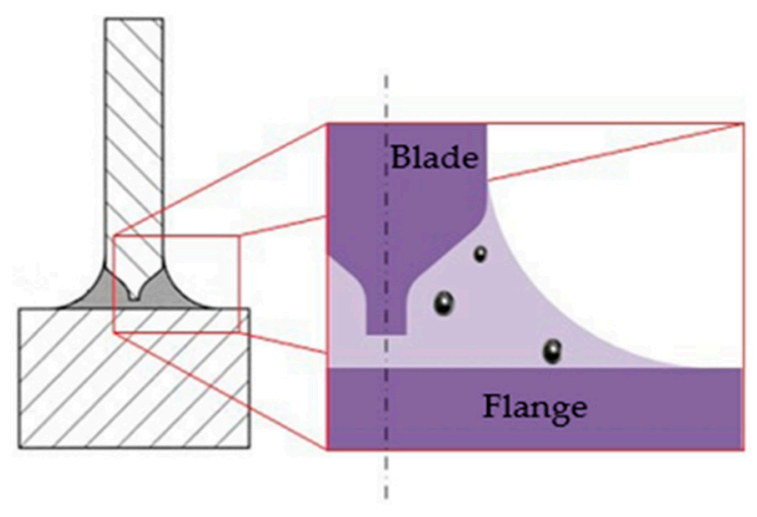

(b) Flaws implementation

Figure 1. The experiment setup overview.

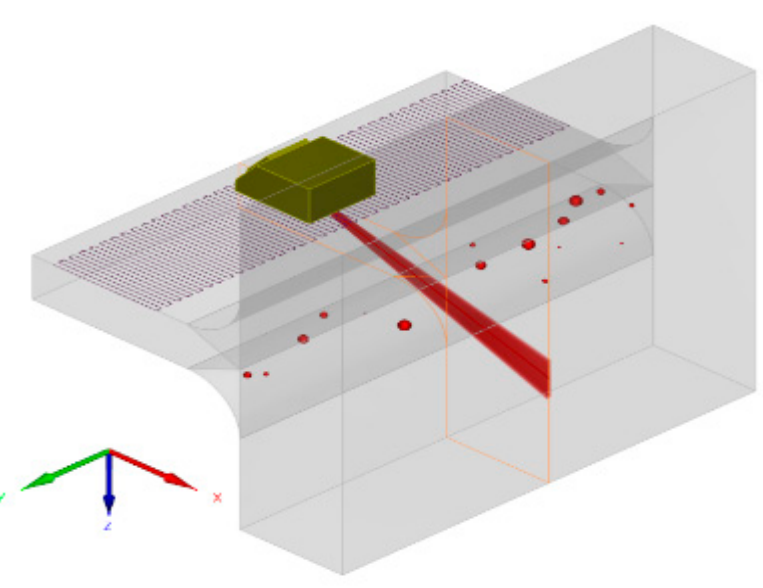

(a) Raster scanning from both blade surfaces

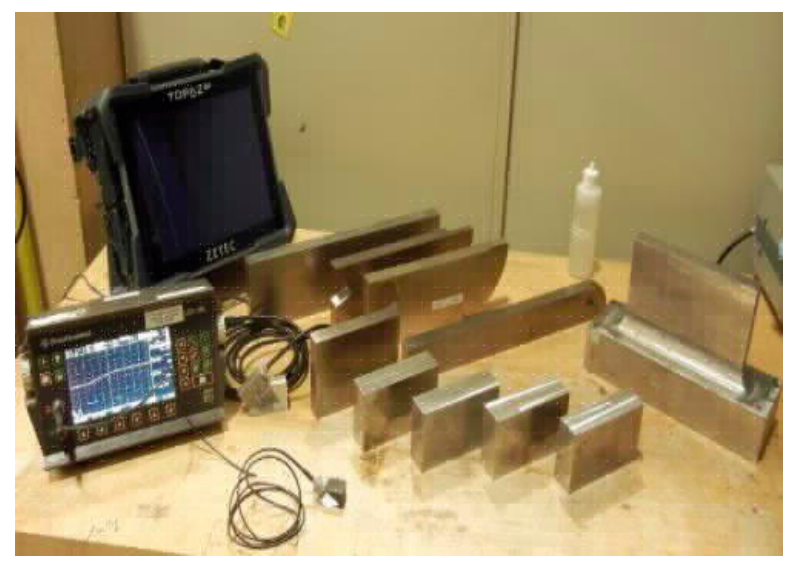

(b) Ultrasonic Testing Equipment

Figure 2. Simulation and experimental Setup.

\subsection{Phased Array Ultrasonic Testing (PAUT)}

The scan plans prepared to inspect the regions of interest were devised according to standards. These scan plans indicate the required refracted beam angle and the probe standoff positions to ensure appropriate volume coverage. Wherever possible, welds shall be inspected from both sides of the blade [19-21]. The ultrasonic system was calibrated using standard blocks to ensure the repeatability and reliability of experimental data $[18,21]$.

Phased Array (PA) transducers can be referred to as multiple small conventional transducers (elements) that are housed within a single unit [22]. Each transducer can be pulsed individually; by varying the delay between elements, the individual waves' constructive interference will occur at the desired angle, which enables the focus to be achieved. The phased array technique is a beamforming method that relies on emission delay to shape the beam in terms of refracted angle and position of the near field. A set of delays required to create a specific beam angle and focus point is called a focal law [23-26]. By electronically changing the delays rapidly, the focal law group can produce an azimuthal scan with a starting angle, a final angle, and an angle step. For example, a focus group can use the following set of angles: starting at $40^{\circ}$, an increment of $1^{\circ}$, and stop at $70^{\circ}$. A phased array probe has limits for beam formation; hence, both wave physics and array limitation must be taken into account. In the experiment, the wedge nominal refracted angle is 55 shear wave and the array of $5 \mathrm{MHz}$, with $0.6 \mathrm{~mm}$ element spacing, produces an acceptable beam profile in the range of $40^{\circ}$ to $70^{\circ}$ shear wave [27-29]. The PAUT inspection was carried out using TOPAZ 64, as shown in Figure 2. The transducers were all linear Phased 
Array Ultrasonic probes operating at a center frequency of $5 \mathrm{MHz}$. The three models used in these inspections differ mainly in terms of the number of elements in the array, namely 12, 16, and 64 (Table 1). They were all used to perform manual or linear-encoded sectorial (Azimuthal) scans ranging from $40^{\circ}$ to $70^{\circ}$ from normal incidence, as shown in Figure 3. Data acquired along two different scan indexes (probe standoff positions) were combined to cover the whole volume of interest in a single file to analyze.

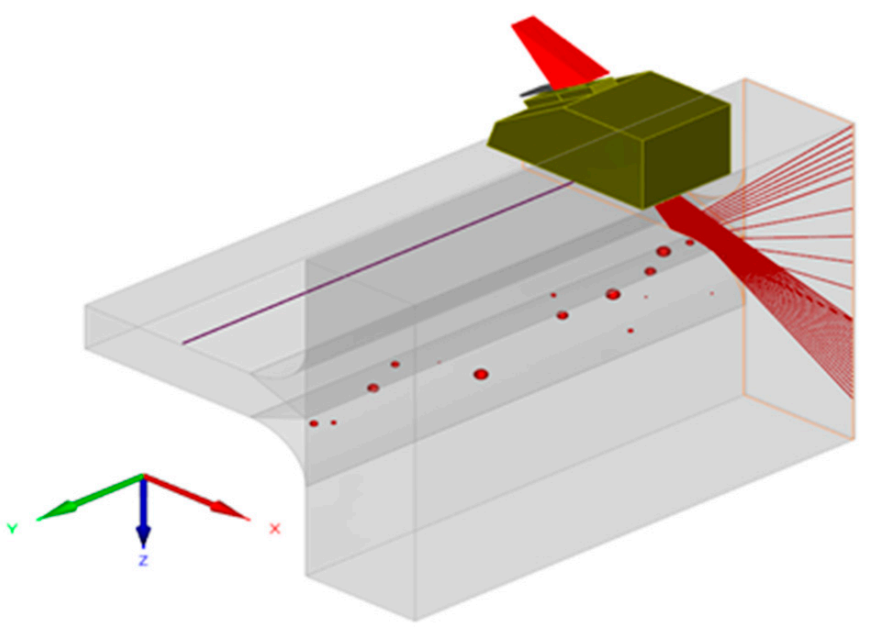

Figure 3. Sectorial scanning from both blade surfaces.

Table 1. Used PAUT probes.

\begin{tabular}{cccccc}
\hline Probe & $\begin{array}{c}\text { Frequency } \\
\mathbf{( M H z )}\end{array}$ & \# Element & $\begin{array}{c}\text { Aperture } \\
\mathbf{( m m )}\end{array}$ & $\begin{array}{c}\text { Elevation } \\
(\mathbf{m m})\end{array}$ & $\begin{array}{c}\text { Wedge } \\
\text { Angle }\left(^{\circ}\right)\end{array}$ \\
\hline 5L12-51S & 5 & 12 & 7.2 & 10 & $51 \mathrm{SW}$ \\
AM5M-55S & 5 & 16 & 9.6 & 10 & $55 \mathrm{SW}$ \\
LM5M-55S & 5 & 64 & 38.4 & 10 & $55 \mathrm{SW}$ \\
\hline
\end{tabular}

The study developed an inspection configuration in accordance with the number of transducer elements and the focal group's capability to insonify the whole weld volume on the opposite face. The inspection strategy involves inspecting the direct skip to prevent beam spread and avoid ghost echoes caused by reflection from the weld cap's curved radius. With a direct skip inspection, the ultrasonic configuration is simpler by mainly focusing the beam for a metal path range of 10 to $50 \mathrm{~mm}$. These permit the use of 16 elements per focal law and use small footprint wedges or a 64-element transducer's front elements to minimize the standoff distance. With these configurations, the focal group's high angle can hit the T-joint center where it meets with the web, and the lower angles can probe most of the weld cap radius. Only a small hidden area exists and cannot be covered with angle beam inspection from the blade. The only inspection technique that can provide 100\% coverage of the weldment is from the web using a normal incidence wedge. Inspection from the web was not attempted because this surface is not accessible during an in-service inspection.

The following conclusions can be drawn from the results obtained;

(1) Flaw resolution increases with the number of active elements used in the array.

(2) However, the use of 32-element does not improve inspection performance. This finding could be attributed to the impact of a larger active aperture on the generated ultrasonic beam's characteristics. Indeed, the beam's exit point is further away from the weld's edge in this case. Besides, the beam is larger, and its power is spread over a larger cross-section, thus reducing the echo reflected by a given flaw. Therefore, focusing has been added to this configuration to narrow down the beam size in the range of interest. 
(3) Linear-encoded PAUT scans can distinguish two closely spaced flaws more easily than manual inspection. You can see example of PAUT results in Figure 4.

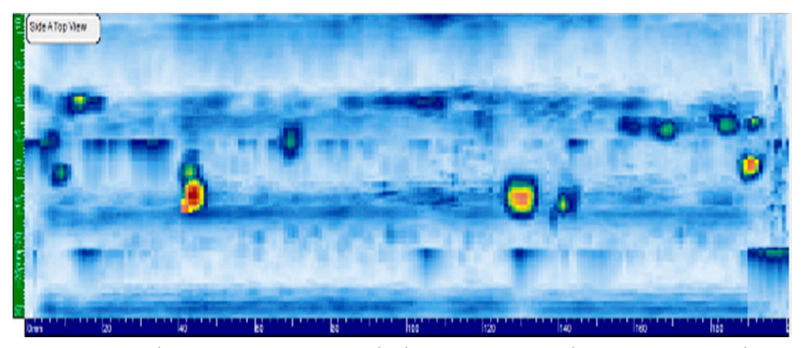

5L12 (12-element): Merged data (-25 and -40 $\mathrm{mm}$ index)

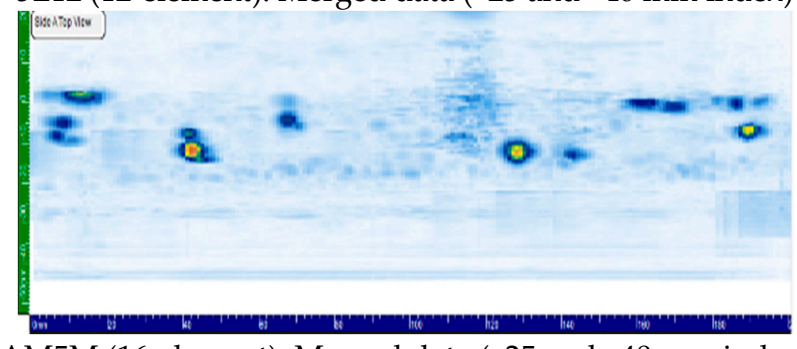

AM5M (16-element): Merged data (-25 and -40 mm index)

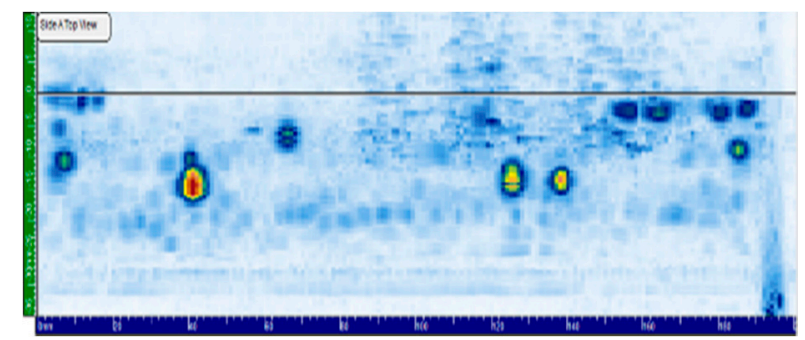

LM5M (16-element): Compound scan (45-64) at -25 mm index

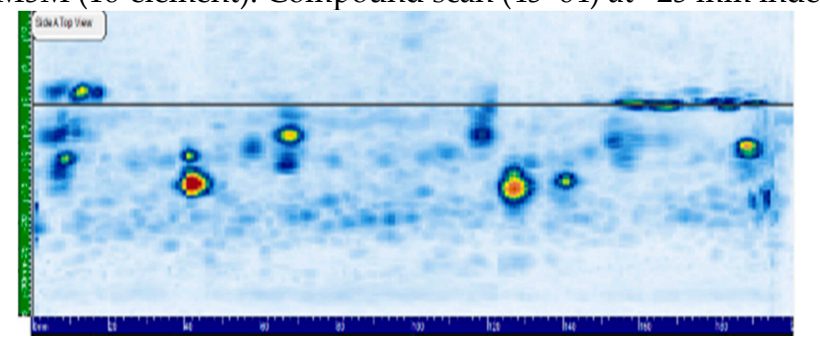

LM5M (32-element): Scan at -25 $\mathrm{mm}$ index with $22 \mathrm{~mm}$ deep focus

Figure 4. Example of PAUT results (Side A, Side view).

\subsection{Total Focusing Method (TFM)}

Total Focusing Method (TFM) is still part of the Ultrasonic Testing (UT). This is because it uses the same basic principles as PAUT as well as the conventional UT [29,30]. In this study, the new phased-array technique called the TFM is included in the test matrix. Unlike the sectorial scan described previously, this technique is not a beamforming method. The TFM is an imaging technique that processes elementary A-scan data to extract the amplitude for a determined path from a firing to a receiving element [31]. After multiplexing all elements as emitter and receiver and delaying and summing all the required amplitude, a data point in the region of interest is assigned an amplitude. The algorithm is simple, but the memory and processing burden increases geometrically with the increase in the number of elements of an array and the size of the area of interest. As a result, it is simple to achieve the configuration of TFM because once the transducer, the wedge, and the mode are defined, the user creates a rectangular area of interest, which is subdivided into a grid. In this regard, the grid spacing is the critical variable, which is normally smaller than one-fifth of the wavelength. However, if the spacing is above this value, the measured amplitude fails to be within $2 \mathrm{~dB}$ of the true amplitude, hence, producing unreliable results. All experiments respected the wavelength criteria and opted for values that had a spacing of at most one-tenth of the wavelength $[28,29,32]$.

The TOPAZ 64 includes a beam simulation amplitude map function that helps to optimize all the parameters. Therefore, with the use of TFM, the region of interest covered the whole weld on the opposite side of the entry surface [29]. In this regard, the sample was to be tested from both sides of the blade in order to fully cover the weldment. The experimental work used a 64-element linear array probe at a nominal frequency of $5 \mathrm{MHz}$. The study used two wedges, one for generating shear wave and one for generating compression wave and performed both manual and encoded scans. The linear encoder scan is done with the wedge's front abutting the weld cap, which produces a scan index of $-25 \mathrm{~mm}$. For manually delivered inspection, the technician has the freedom to make transversal, lateral, and skewing movements to locate discontinuities and peak them. The T-joint was examined using T-T and L-L direct modes with linear encoded scans and manual inspection. The T-T and L-L modes were used in the experiments because they produce a good amplitude response from a volumetric discontinuity. In this study, 
before starting the sample's inspection, the $3 \mathrm{~mm}$ SDH was used on the martensitic sample (same material properties) for TFM model calibration. From these results, we obtained the following conclusions;

1. TFM results appear clearer, with fewer spurious indications and better-defined flaw geometries.

2. The Longitudinal-Longitudinal reconstruction mode seems to give slightly better results than the Transverse-Transverse mode.

3. We obtained a slightly better detection rate with manual TFM as compared to the encoded one. We suspect that this is due to the operator's ability to minutely adjust the probe's position to improve the signal to noise ratio on the testing unit display. An example of TFM results (with two different reconstruction modes) compare with 32-element PAUT result is observed in Figure 5.

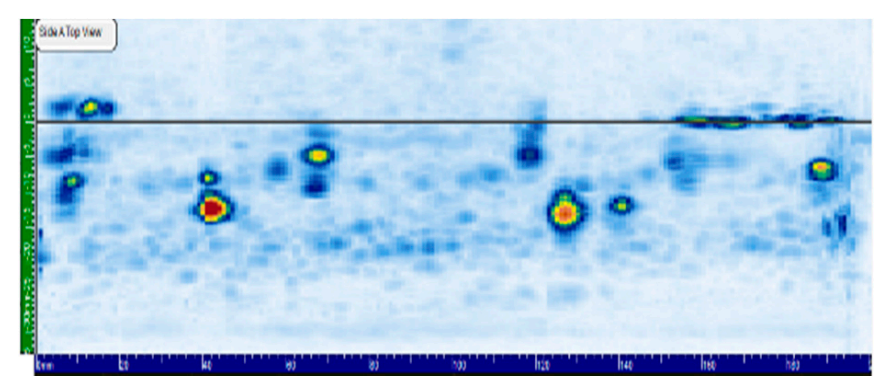

(a) LM5M (32 elements): Sectorial scan at $-25 \mathrm{~mm}$ index with $22 \mathrm{~mm}$ deep focus.

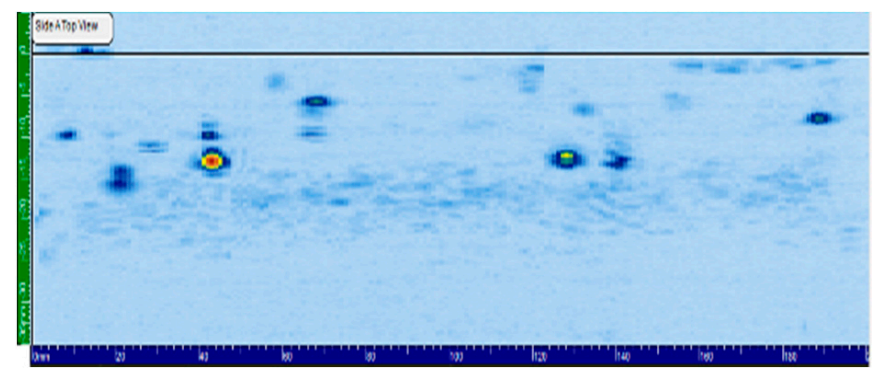

(b) LM5M (64 elements): FMC scan at $-25 \mathrm{~mm}$ index with encoder, direct TFM T-T.

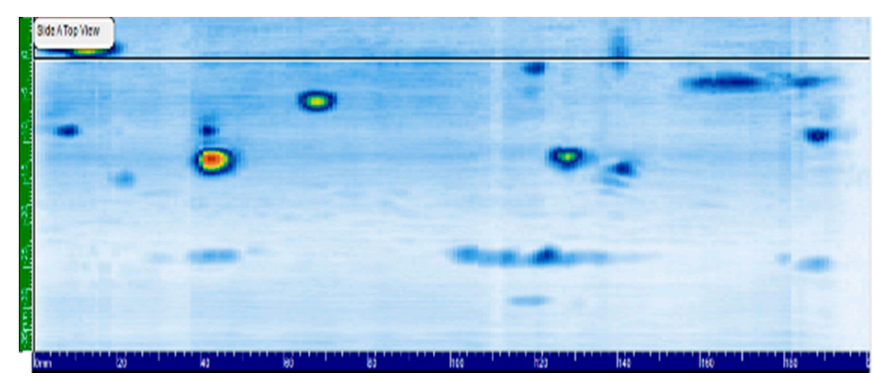

(c) LM5M (64 elements): FMC scan at $-25 \mathrm{~mm}$ index with encoder, direct TFM L-L.

Figure 5. Example of TFM results (Side A, side view).

\subsection{Experimental Detection Rate}

The performance of the different ultrasonic inspection techniques tested are compared in Figure 6. Obviously, among all these techniques, conventional UT results have the lowest detection rate. This could be because the fixed-angle beam can miss a flaw more easily than with ultrasounds impinging the flaw from different angles. Although the inspection is carried out to a well-defined standard, the detection rate is highly dependent on the operator. The certified practitioners reported between 7 to 16 flaws out of the 30 embedded in the weld, with an average reporting of 11 flaws. 
90

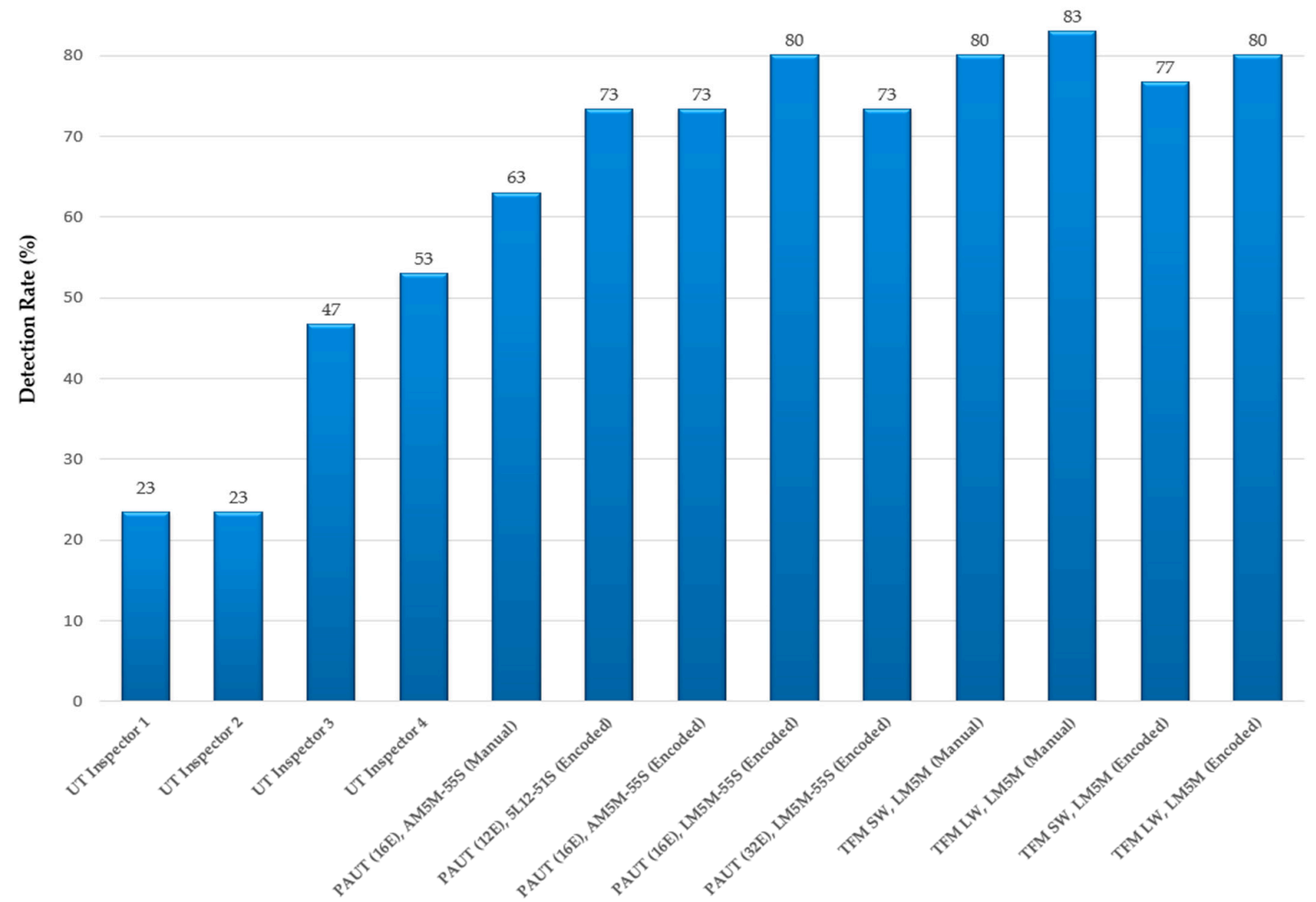

Experiment, Different U1trasonic Methods

Figure 6. Experiment, Different ultrasonic methods.

Several difficulties were observed with manual testing; (a) Challenging to report a flaw nearby a larger reflector with all the attention on the large reflector, (b) Manual reporting of flaw position is cumbersome and leads to errors, (c) Low amplitude signal just above the rejection threshold is easily discarded, (d) the report's quality is directly related to the inspector's diligence in taking clear and precise notes. If the report is written several days after the test, the notes' quality is of paramount importance [33].

Manual phased array inspection suffers some of the limitations observed with a fixed-angle beam probe. The detection is done on the fly, and the flaw's information is written during the inspection. If the sample has several flaws, the technician can get confused when measuring and reporting flaws. However, one of the advantages of manual PAUT inspection is the freedom the operator has to explore a suspected flaw by executing various probe motion; pivoting the transducer or orbital movement to reveal the target's volumetric features. The manual phased-array inspection provided the highest maximum amplitude measured and revealed flaws hidden in the trailing edge weld reinforcement. However, even if the manual phased-array found flaws hidden in the geometry, on average, it detected fewer flaws than the linear-encoded phased array.

The linear encoded inspection provides repeatable scans, and flaws with low amplitude are detected more readily by comparing the local base noise to check for any localized increase that would reveal a flaw. Close flaws may be separated, and when using the projection views, their location and separation can be measured. Generally, the aperture of 12 and 16 elements produced a reasonably good detection rate, and the small footprint 
of the 12-element transducer allows it to cover the center of the weld. As the aperture increases, the stand-of distance increases, and the weld's center is no longer covered. This explains why the 32-element tests did not perform better than the 12 or 16 element tests despite having a smaller beam diameter and a more extended working range covering the whole weld path.

The TFM method produced a higher detection rate, slightly better than a sectorial scan. One advantage noticed during the test is the operator can readily size the diameter of the flaw. This helped identify small beads from noise since the shape was maintained while probing the area. The TFM could also probe nearer to the surface and provided good coverage of the whole weldment. There is no dead zone compared to a 32 element sectorial scan. In our sample, the material has a low noise level suitable for a TFM inspection, which explains the high detection rate obtained with the study sample. Another advantage of the TFM is flaws could be categorized in apparent size or apparent reflective features. Small ceramic bead produces a specific round-shaped signal area proportional to their size, while larger beads showed either oriented facets or multiple facets surrounding the bead. TFM provided details about the reflective surface's nature, while a sectorial scan did not provide any hints about the flaw's small features. A multi-element probe is, therefore, a better candidate in terms of detection rate. Although the difference is not huge, TFM seems to perform slightly better than standard PAUT sectorial scanning.

The optimum inspection strategy in order to reveal the most implanted flaws is to perform the inspection in two steps:

Step 1: linear encoded sectorial scan with an aperture of 16 elements or linear encoded TFM scan.

Step 2: manual inspection of the trailing edge and weld reinforcement using either a sectoral scan with an aperture of 16 elements or a 64-element TFM scan. In practice, because a real runner's trailing edge is not a flat surface, a small PAUT probe with a 12 or 16- element aperture is the only practical method for that region.

\section{CIVA Modelling and Analysis}

The current study used the ultrasonic module of CIVA to simulate the welded T-joint sample inspection considered previously. Models for the sample and the inspection system have been defined as accurately as possible. There is a wide range of input parameters available to the model (Figure 7) [34]; only to give a few of the different parameters that need to be entered, we can mention:

- a CAD model describing the dimension of the sample.

- ultrasonic properties of the steel used to manufacture the sample.

- the location, the size, and the nature of the flaws distributed in the welded joints of the sample.

- ultrasonic probe geometry including a description of the array settings required for PAUT.

- the firing sequence of the elements as well as a description of the exciting impulse.

However, before embarking on a campaign of numerical simulations, it is necessary to validate the various parameters entered through measurements on real samples. This step was performed with a standard calibration block manufactured from the same steel alloy. The study carried out all the steps under the International Institute of Welding [35-37].

\subsection{Simulation of Conventional Ultrasonic Testing (UT)}

A rectangular single element crystal assembled to simulate conventional ultrasonic testing to a flat refracting wedge was selected as the probe. Both beams refracting at $60^{\circ}$ and $70^{\circ}$ were tested, as shown in Figure 8. Two steel alloys with slightly different ultrasonic properties have been used for the T-joint sample model. Spherical voids, whose distribution and size mirror the manufactured T-joint sample, have been inserted into the model, as shown in Figure 9. In the real sample, the flaws were produced by silicon nitride balls whose ultrasonic impedance is very similar to that of steel. However, experimentally, 
we have noticed that ultrasonic reflection does not come from an impedance mismatch at the steel/ceramic interface but from a lack of wetting between the two. Therefore, for simulation purposes, we prefer to use voids. According to Hydro-Quebec's procedure, the numerical system's sensitivity was calibrated, namely, a side-drilled-hole with a diameter of $1.5 \mathrm{~mm}$ at a depth of $20 \mathrm{~mm}$.

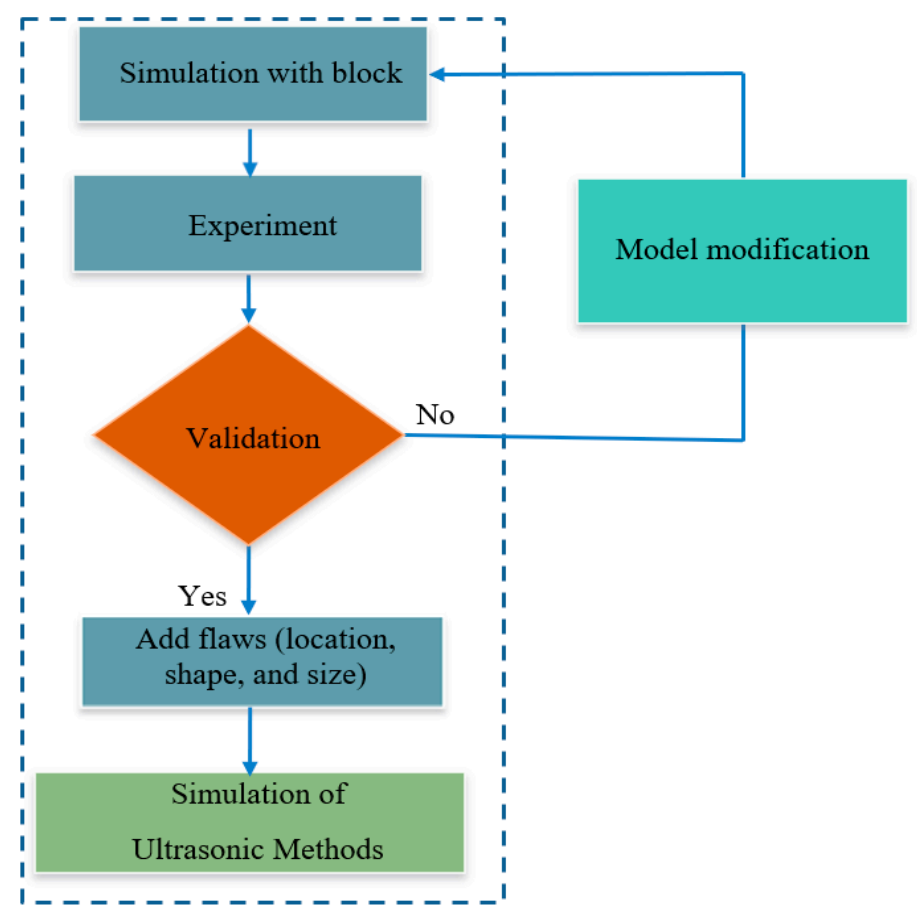

Figure 7. Configuring the CIVA model and input parameters.

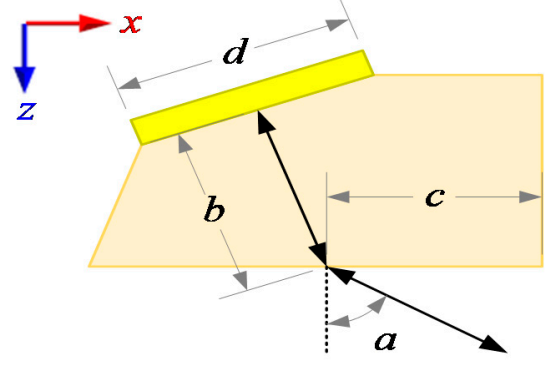

(a) Conventional ultrasonic testing probe

\begin{tabular}{|cccc|}
\hline$a\left(^{\circ}\right)$ & $b(\mathrm{~mm})$ & $c(\mathrm{~mm})$ & $d(\mathrm{~mm})$ \\
\hline 60 & 20 & 18 & 16 \\
\hline 70 & 20 & 15 & 16 \\
\hline
\end{tabular}

(b) The dimension of the probe

Figure 8. Parameters of the ultrasonic probe.

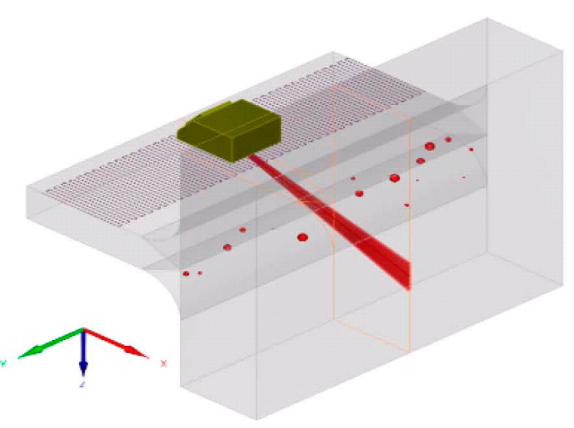

(a) Raster scanning with water coupling

\begin{tabular}{|ccc|}
\hline Velocity & UNS S41500 & Alloy steel \\
\hline Longitudinal wave $\left({\left.\mathrm{m} . \mathrm{s}^{-1}\right)}^{-1}\right.$ & 5880 & 5920 \\
\hline Transverse wave $\left(\mathrm{m}^{-1}\right)$ & 3210 & 3255 \\
\hline Density $\left(\mathrm{g} . \mathrm{cm}^{-3}\right)$ & 7.9 & 7.8 \\
\hline
\end{tabular}

(b) Material Properties

Figure 9. Scanning pattern and material properties. 
The following conclusions were obtained from the simulation results: Sample alloys appear to have little to no impact on the system's detection rate and could probably be attributed to the similarity between the two alloys' ultrasonic properties. Further work will consider the impact of steel microstructure on the ultrasonic beam's scattering, increasing its attenuation and producing a higher background noise level. Beam refracted at $70^{\circ}$ gave a better detection rate than beam refracted at $60^{\circ}$. This result agrees with our experimental measurements and tends to prove our hypothesis; namely, this performance is due to the beam's larger divergence. However, we observe a significant difference between the simulated detection rate (Figure 10) compared to that obtained experimentally (Figure 6). The reasons for this difference are that the ceramic beads do not behave exactly like a void, and the simulation does not capture the operator's judgment.

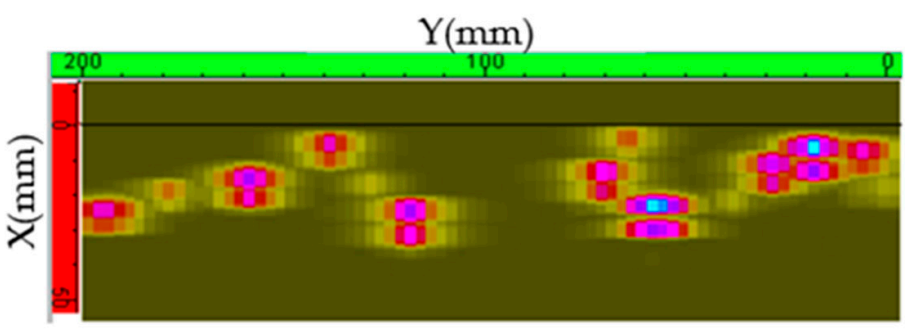

(a) $70^{\circ}$ refracted beam

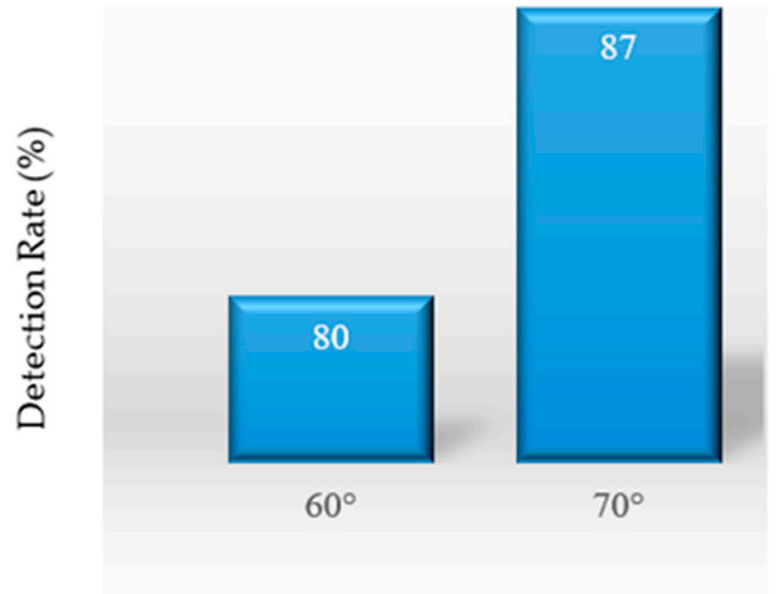

(b) Simulated detection rate for Alloy steel and Martensitic stainless steel by probe $60^{\circ} \& 70^{\circ}$

Figure 10. Simulation results from Conventional ultrasonic testing probe.

\subsection{Simulation of Phased Array Ultrasonic Testing (PAUT)}

A linear array of 64 elements assembled to a flat refracting wedge was selected as the probe to simulate phased array ultrasonic testing. In all cases, this probe was used to sectorial scan the sample at angles ranging from $40^{\circ}$ to $70^{\circ}$ (with \& without focusing) as shown in Figure 11. If there was focusing, the focal depth was either 22 or $50 \mathrm{~mm}$. The electronic focusing enables optimizing the beam shape at the expected defect location and further optimizes the detection probability. Also, focusing at multiple depths improves the ability to size critical flaws for volumetric inspections.

The T-joint sample model was the same as the one used for conventional UT simulation; same material options, same flaws nature, size, and distribution. According to HydroQuebec's procedure, the numerical system's sensitivity was calibrated, namely three sidedrilled-holes with a diameter of $1.5 \mathrm{~mm}$ at depths of 15, 30, and $45 \mathrm{~mm}$, respectively. The study used the computed time corrected gain (TCG) in this calibration process.

The following conclusions were obtained from the simulation results: Sample alloys appear to have little to no impact on the detection rate of the system; A 16-element configuration has a better detection rate than a 32-element one (Figure 12). The result agrees with the experimental measurements and tends to prove our hypothesis, namely, the impact of the beam exit point and the width of the refracted beam on the sensitivity of these techniques; by phased-array sectorial scanning, we are sweeping the volume with a variety of angles. Even if the targets are not exactly aligned with the center ray as indicated by CIVA, there will be components of the wavefront that might hit the targets with a preferred reflection coefficient and directivity. Of course, the S-scan's fixed standoff 
is a limiting factor that could cause flaws at the extremes of the sectorial sweep to have a weaker response. This will be a factor in the way we set up the 16 element and 32 element apertures; for the same refracted angle, the 32 element aperture has an exit point farther back from the weld. So we are approaching the targets from slightly different angles (and distances).

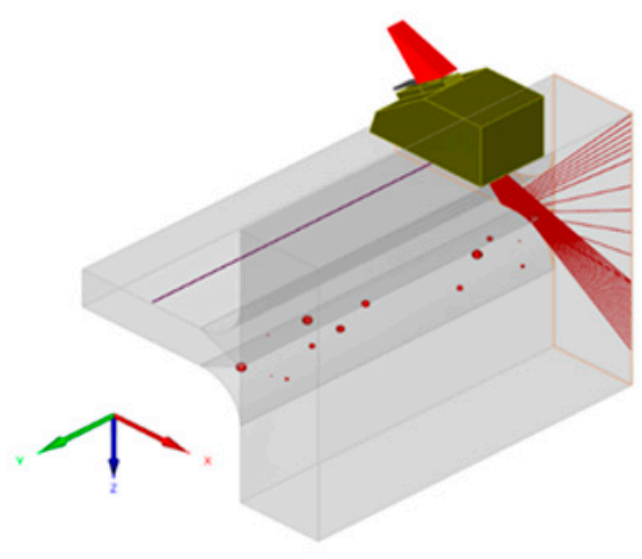

(a) Raster scanning with water coupling

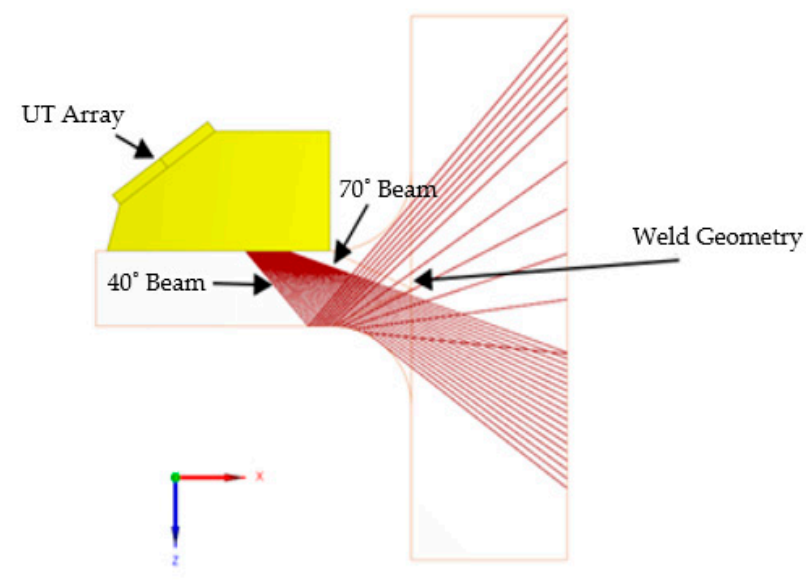

(b) Setup CIVA ray tracing with the selected sweep angles.

Figure 11. CIVA Simulation Configuration.

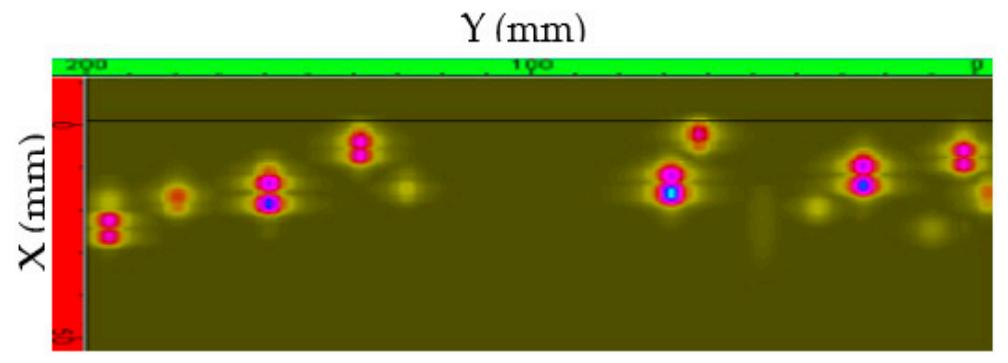

(a) 16-element, without focusing

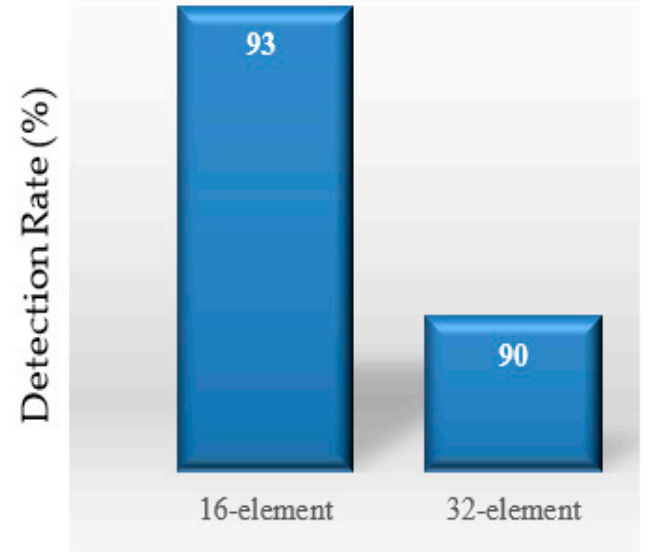

(b) Simulated detection rate for Alloy steel and Martensitic stainless steel by 16 \& 32 element

Figure 12. Simulation results from PAUT probe.

PAUT simulated detection rate is significantly better than the conventional UT one. As mentioned previously, this could be because the fixed-angle beam can miss a flaw more easily than with ultrasounds impinging the flaw from different directions, as is the case with sectorial scans. Also, with a fixed angle and using a raster scan, detection will be a variable that can be a function of the wedge size (limiting the probe's access to the weld) and the beam divergence.

\section{Finding and Discussion}

Our tests showed that conventional Ultrasonic results in the lowest detection rate among the different ultrasonic testing methods evaluated. Although the inspection is carried out to a well-defined standard, the detection rate is highly dependent on the 
operator. Fixed angle conventional ultrasonic testing (UT) provides less information for accurate assessment of actual flaw conditions. Detection capability increases with the divergence of the beam of a single element probe. Because a beam refracted at $70^{\circ}$ is more likely to show greater divergence than one refracted at $60^{\circ}$, the former has a better detection rate. PAUT allows one to use more angles and increases the probability of detection compared to conventional UT. PAUT sectorial scan is also well adapted to the T-joint sample's geometry. Low angles will be tangent to the weld cap radius, while the high angles will probe the center of the blade to the web region.

The 16-element PAUT probe proves to be a good compromise for detection: Better resolution than the 12-element probe; Compared to the 32-element, a smaller wedge can be used, and for the same refracted angle, the beam exit point is closer to the edge of the weld; The size of the beam is adapted to the range of interest. With 32-element, focusing is needed to narrow down the beam emitted from a larger active aperture but did not lead to a better detection rate. Linear-encoded PAUT scans can distinguish two closely spaced flaws more easily than manual inspection.

Among the different ultrasonic testing methods evaluated, manual TFM resulted in the highest detection rate. It is slightly better than encoded TFM due to the operator's ability to adjust the probe's position to improve the signal to noise ratio on display. TFM $\mathrm{L}-\mathrm{L}$ reconstruction mode seems to give slightly better results than the T-T reconstruction mode. The reason for such a result is still under investigation. TFM by nature has better coverage than beam-forming PAUT, and that could explain the slightly higher detection rate. TFM has the advantage of a clear display with appropriate information on the actual distribution and geometry of the flaws (faceted, multi-faceted, sphere-like). However, using the T-T mode did provide more details of the ceramic bead interface and better estimate of the diameter because of the shorter wavelength. TFM requires a transducer with a large number of elements. Thus, a large contact area and good coupling for all the ray paths are needed to produce consistent and repeatable results.

The best inspection strategy to inspect the trailing edge of a runner blade is to perform the inspection in two steps:

Step1: Linear encoded scan with either PAUT sectorial scanning or TFM direct mode. PAUT shall use shear wave with the refracted angle from $40^{\circ}$ to $70^{\circ}$. TFM could use either L-L or T-T direct mode.

Step 2: Manual sectorial scanning with a 12 or 16-element aperture and a small wedge footprint to inspect the trailing edge and the weld reinforcement. The small footprint wedge is essential on a real turbine because of the trailing edge's curvature to maintain good contact while scanning.

\section{Conclusions}

The preliminary technical approach developed in phase I of this research was tested on a T-weld specimen. When developing the scan plans and the preliminary data analysis, the ray path analysis indicated the need to carry out a minimum of two scans at different index points to enable complete volume coverage of the weld, particularly for thick weld specimens. This study aimed to collect initial data on a simplified mock-up and then compare the experimental ultrasonic data with the results of simulations performed by CIVA. Experimental studies in a laboratory setup showed that, among the different ultrasonic testing methods evaluated, TFM appears to be the best ultrasonic inspection technique to provide reliable flaw data to the IREQ's life estimation model. In addition to this technique's ability to detect flaw distribution, the algorithm allows a better definition of flaw geometry and highlights its different facets. CIVA model could be used to simulate ultrasonic inspection of a weld. However, to obtain reliable data, the sample and the inspection system's models must be defined with a high accuracy level. To do this, we need to adjust model parameters through validation loops based on precise measurements on real calibration samples. While defining accurate numerical models seems like a tedious task, it ultimately drastically reduces the costs of developing a reliable inspection strategy. 
Our future work uses such models to estimate the probability of detection (POD) of certain ultrasonic techniques applied to hydroelectric turbine runners' inspection.

Author Contributions: Conceptualization, M.E.B.; Formal analysis, M.E.B.; Funding acquisition, D.T.; Investigation, G.R. and M.V.; Methodology, M.E.B.; Software, M.E.B.; Supervision, M.V.; Validation, G.R.; Writing-original draft, M.E.B.; Writing-review \& editing, G.R. and M.V. All authors have read and agreed to the published version of the manuscript.

Funding: This study is financially supported under the MITACS accelerate program in collaboration with the Institut de recherche d'Hydro-Quebec (IREQ). The corresponding author is also financially supported by the "oN Duty!" program. oN DuTy! is an initiative developed under the Collaborative Research and Training Experience (CREATE) of the National Sciences and Engineering Research Council of Canada (NSERC). (http:/ / www.ondutycanada.ca/ (accessed on 13 May 2021)).

Institutional Review Board Statement: Not applicable.

Informed Consent Statement: Not applicable.

Data Availability Statement: Not applicable.

Acknowledgments: We would like to deeply thank IREQ for their kind support. We are also grateful to Quebec Metallurgy Center (CMQ) for their valuable advice and support during experiments, in particular Jeremy carignan. The authors also wish to acknowledge Edward Ginzel, Martin Gagnon, and Hamid Habibzadeh Boukani for many discussions and their technical assistance.

Conflicts of Interest: The authors declare no conflict of interest. The funders had no role in the design of the study; in the collection, analyses, or interpretation of data; in the writing of the manuscript, or in the decision to publish the results.

\section{References}

1. Huertas-Hernando, D.; Farahmand, H.; Holttinen, H.; Kiviluoma, J.; Rinne, E.; Söder, L.; Milligan, M.; Ibanez, E.; Martínez, S.M.; Gomez-Lazaro, E. Hydro power flexibility for power systems with variable renewable energy sources: An IEA Task 25 collaboration. Wiley Interdiscip. Rev. Energy Environ. 2017, 6, e220. [CrossRef]

2. Boukani, H.H.; Viens, M.; Tahan, S.-A.; Gagnon, M. Case study on the integrity and nondestructive inspection of flux-cored arc welded joints of Francis turbine runners. Int. J. Adv. Manuf. Technol. 2018, 98, 2201-2211. [CrossRef]

3. Boukani, H.H.; Viens, M.; Tahan, S.A.; Gagnon, M. On the performance of nondestructive testing methods in the hydroelectric turbine industry. In IOP Conference Series: Earth and Environmental Science; IOP Publishing: Bristol, UK, 2014; Volume 22, p. 12018.

4. Gagnon, M.; Tahan, A.; Bocher, P.; Thibault, D. A probabilistic model for the onset of High Cycle Fatigue (HCF) crack propagation: Application to hydroelectric turbine runner. Int. J. Fatigue 2013, 47, 300-307. [CrossRef]

5. Bray, D.E.; Stanley, R.K. Nondestructive Evaluation: A Tool in Design, Manufacturing and Service; CRC Press: Boca Raton, FL, USA, 1996; ISBN 0849326559.

6. ASTM E1316-13a. Standard Terminology for Nondestructive Examinations; ASTM International: West Conshohocken, PA, USA, 2013.

7. Cheeke, J.D.N. Fundamentals and Applications of Ultrasonic Waves; CRC Press: Boca Raton, FL, USA, 2017 ; ISBN 1351833197.

8. Shull, P.J. Nondestructive Evaluation: Theory, Techniques, and Applications; CRC Press: Boca Raton, FL, USA, $2002 ;$ ISBN 0203911067.

9. Felice, M.V.; Fan, Z. Sizing of flaws using ultrasonic bulk wave testing: A review. Ultrasonics 2018, 88, 26-42. [CrossRef] [PubMed]

10. Ahmad, A.; Bond, L.J. Fundamentals of Ultrasonic Inspection. ASM Int. 2018, 17, 155-168.

11. Zhang, Y.; Li, X.; Zhang, J.; Ding, H. Model based reliability analysis of PA ultrasonic testing for weld of hydro turbine runner. Procedia Eng. 2011, 16, 832-839. [CrossRef]

12. Xiao, K.; Shi, Y.K.; Ma, Q.Z.; Zhang, J.; Li, X.H. The intelligent ultrasonic system for quality testing of weld connections in turbine runners. In Advanced Materials Research; Trans Tech Publications Ltd.: Stafa-Zurich, Switzerland, 2013; Volume 774, pp. 1543-1546. [CrossRef]

13. Zhang, J.; Li, X.; Shi, Y.; Liang, L. Phased array ultrasonic inspection of embedded defects in hydropower turbine runner welds. Insight-Non-Destr. Test. Cond. Monit. 2014, 56, 390-394. [CrossRef]

14. Katchadjian, P. Practical applications of ultrasonic testing in nuclear and conventional industry. Insight-Non-Destr. Test. Cond. Monit. 2004, 46, 754-757. [CrossRef]

15. Habibzadeh Boukani, H. Evaluation of the Reliability of Nondestructive Ultrasonic Inspection Methods for the Detection and the Characterization of Defects in Hydroelectric Turbine Welded Joints. Ph.D. Thesis, ETS University, Montreal, QC, Canada, 2018.

16. Gagnon, M.; Tahan, A.; Bocher, P.; Thibault, D. Influence of load spectrum assumptions on the expected reliability of hydroelectric turbines: A case study. Struct. Saf. 2014, 50, 1-8. [CrossRef]

17. Zhang, X.; Zhang, Y.; Tian, B.; An, J.; Zhao, Z.; Volinsky, A.A.; Liu, Y.; Song, K. Arc erosion behavior of the Al2O3-Cu/(W, Cr) electrical contacts. Compos. Part B Eng. 2019, 160, 110-118. [CrossRef] 
18. Canadian Standards Association (CSA). CSA W59-13 Welded Steel Construction (Metal Arc Welding); Canadian Standards Association (CSA): Mississauga, ON, Canada, 2013; ISBN 9781771392815.

19. ISO 17640 Non-Destructive Testing of Welds_Ultrasonic Testing_Techniques, Testing Levels, and Assessment; International Organization for Standardization: Geneva, Switzerland, 2018.

20. ISO 13588 Non-Destructive Testing of Welds, Ultrasonic Testing, Use of Automated Phased Array Technology; International Organization for Standardization: Geneva, Switzerland, 2019.

21. ASTM E2700-14 Standard Practice for Contact Ultrasonic Testing of Welds Using Phased Arrays; ASTM International: West Conshohocken, PA, USA, 2014.

22. Drinkwater, B.W.; Wilcox, P.D. Ultrasonic arrays for non-destructive evaluation: A review. NDT e Int. 2006, 39, 525-541. [CrossRef]

23. Phased Array Ultrasonic Testing (PAUT)-Welds. Available online: https://www.twitraining.com/home/programmes-andcourses/non-destructive-testing/advanced-ndt-methods/phased-array-ultrasonic-testing-welds (accessed on 20 May 2021).

24. ISO 19675 Non-Destructive Testing-Ultrasonic Testing—Specification for a Calibration Block for Phased Array Testing (PAUT); International Organization for Standardization: Geneva, Switzerland, 2017.

25. Phased Arrzay Testing: Basic Theory for Industrial Applications. Available online: https://www.yumpu.com/en/document/ $\mathrm{read} / 3700722$ / phased-array-testing-basic-theory-for-industrial-olympus (accessed on 20 May 2021).

26. Ginzel, E.A.; Johnson, D. Phased-Array Resolution Assessment Techniques. E-J. Nondestruct. Test. 2008. Available online: https:// www.eclipsescientific.com/papers/Phased-Array\%20Resolution\%20Assessment\%20Techniques.pdf (accessed on 13 May 2021).

27. Ginzel, E. Phased Array Ultrasonic Technology; Eclipse Scientific Products Incorporated: Waterloo, ON, Canada, 2013; ISBN 9780991709502.

28. Ditchburn, R.J.; Ibrahim, M.E. Ultrasonic Phased Arrays for the Inspection of Thick-Section Welds; Defense Science and Technology Organization Victoria (Australia) Maritime Platforms Division: Canberra, Australia, 2009.

29. Richard, D.; Zottig, F.; Maes, G. On the Use of Advanced Focusing Techniques for Enhanced PAUT Inspection Capability. In Proceedings of the 12th European Conference on Non-Destructive Testing (ECNDT 2018), Gothenburg, Sweden, 11-15 June 2018.

30. Holmes, C.; Drinkwater, B.W.; Wilcox, P.D. Post-processing of the full matrix of ultrasonic transmit-receive array data for non-destructive evaluation. NDT e Int. 2005, 38, 701-711. [CrossRef]

31. Zhang, J.; Drinkwater, B.W.; Wilcox, P.D. Comparison of ultrasonic array imaging algorithms for nondestructive evaluation. IEEE Trans. Ultrason. Ferroelectr. Freq. Control 2013, 60, 1732-1745. [CrossRef] [PubMed]

32. Principles of Full Matrix Capture (FMC) and Total Focusing Method (TFM) in Ultrasonic Inspections. Available online: https:/ / www.slideshare.net/ZetecNDT/ principles-of-full-matrix-capture-fmc-and-total-focusing-method-tfm-in-ultrasonicinspections (accessed on 20 May 2021).

33. Chaplin, R. Industrial Ultrasonic Inspection: Levels 1 and 2; Friesen Press: Victoria, BC, Canada, 2017; ISBN 1460295684.

34. Cinquin, M.; Lonné, S.; Raillon, R.; Darmon, M.; Mahaut, S.; Le Ber, L. Results of the 2005 UT Modelling Benchmark Obtained with the CIVA Software Developed at the CEA. AIP Conf. Proc. 2006, 820, 1828-1835. [CrossRef]

35. Iakovleva, E.; Chatillon, S.; Bredif, P.; Mahaut, S. Multi-mode TFM imaging with artifacts filtering using CIVA UT forwards models. AIP Conf. Proc. 2014, 1581, 72-79.

36. Calmon, P. Recommendations for the Use and Validation of NDT Simulation. In VIiw Best Practice Document International Institute of Welding (IIW); International Institute of Welding (IIW): Genoa, Italy, 2013; pp. 2313-2363. ISBN 978-2-9541979-2-0.

37. Mahaut, S.; Darmon, M.; Chatillon, S.; Jenson, F.; Calmon, P. Recent advances and current trends of ultrasonic modelling in CIVA. Insight-Non-Destr. Test. Cond. Monit. 2009, 51, 78-81. [CrossRef] 